\title{
The Right Face Mask Detection System using Deep Learning Methods
}

\author{
Bagus Hendra Saputra \\ Departement of Electrical Engineering Master \\ Program, \\ Gunadarma University, Jakarta, Indonesia
}

\author{
Wahyu Kusuma Raharja \\ Departement of Electrical Engineering Master \\ Program, \\ Gunadarma University, Jakarta, Indonesia
}

\begin{abstract}
Coronavirus Disease 2019 (COVID-19) is an emerging respiratory disease caused by acute respiratory syndrome (SARS-CoV-2). As of June 23, 2021, as a result of the virus infected patients reached 178,837,204 and 3,880,450 died. Therefore, WHO (World Health Organization) imposes health protocols that must be implemented by all individuals throughout the country, based on that the government of the Republic of Indonesia through the Decree of the Minister of Health of the Republic of Indonesia Number HK.01.07 / MENKES / 382/2020 concerning health protocols for people in places and public facilities in order to prevent and control corona virus disease 2019 (COVID-19) which is mandatory to wear a mask when out of the house, Maintain a strict social distance in public places of at least 1 meter and often wash hands or sanitize hands with disinfectant.
\end{abstract}

In this research, the researchers create a system to conduct supervision on the community to be able to comply with health protocols, especially the use of appropriate masks. This system is called the right Face Mask Detection System using deep learning methods with MobileNetV2 module and Tensorflow framework.

Based on 90 tests conducted obtained the results of 7 invalid tests, so that the accuracy of the system that has been built has an average of $86.95 \%$.

\section{Keywords}

deep learning, face mask, face detection, mobilenetV2, COVID-19.

\section{INTRODUCTION}

Some people around the world have been affected by the coronavirus (COVID-19). This crippled the economic growth of all nations in the world, and caused some victims to be treated to death. Coronavirus Disease 2019 (COVID-19) is an emerging respiratory disease caused by severe acute respiratory syndrome coronavirus 2 (SARS-CoV-2) [1] As of June 23, 2021, as a result of the virus infected patients reached $178,837,204$ and 3,880,450 died [2]. Therefore, to fight and reduce the transmission and spread of the virus more widely among the community in various countries, WHO (World Health Organization) imposes health protocols that must be implemented by all individuals in all countries, based on that the government of the Republic of Indonesia through the Decree of the Minister of Health of the Republic of Indonesia Number HK.01.07 / MENKES / 382/ 2020 on health protocols for the public in places and public facilities in Indonesia. The prevention and control of corona virus disease 2019 (COVID-19) is mandatory to wear a mask when out of the house, maintain a strict social distance in public places of at least 1 meter and often wash hands or sanitize hands with disinfectant. Research results obtained from several health journals show that wearing masks is important to prevent the spread of the virus [3][4][5][6] Using a mask will effectively minimize the virus that interferes in the air so that it can cause infection of the virus cannot reach into the human respiratory system by filtering the air inhaled by humans. However, the effectiveness of the use of masks in preventing the transmission of the virus in the community is reduced due to education in the community and limited supervision. So, the development of automatic detection in the supervision of the use of masks is very important to provide protection individually and society at large in preventing epidemics locally and globally.

Therefore, to deal with these problems, technology is needed that can help in terms of supervision in the community to be able to comply with health protocols. This technology is called the right Face Mask Use Detection System which is part of Deep Learning and the development of Face Detection. The right face mask detection system aims to recognize faces that use masks or do not use masks as well as the use of masks correctly or not in accordance with applicable rules[7] This precise Face Mask Usage Detection System uses the Tensorflow framework, with the MobileNetV2 module.

\section{LITERATURE REVIEW}

In conducting this research, it is very important to know the references from the previous research. This is done to avoid duplication and as a learning material so that research can continue to grow. Below are some reviews of previous research.

In the study [8] entitled "FACEMASK DETECTION USING CONVOLUTIONAL NEURAL NETWORK", this study

proposed a model resulting from the integration between deep learning and classical deep learning techniques using OpenCV, Tensorflow And Keras. This model consists of 2000 images divided by the sharing of images that use masks and images that do not use masks. The images were then used as datasets that were then used to build models of the Convolutional Neural Network (CNN). The model, built using CNN, aims to identify everyone whether or not to use a mask from an image or video stream. In order to achieve this goal, steps are needed in the process of making the $\mathrm{CNN}$ model contained in the following methodology:

1. Data Visualization: This step aims to visualize images in datasets so that they can be easily understood and the exchange of information can run well and efficiently

2. Data Augmentation: This step aims to prepare datasets for training in order to get better model performance.

3. Create a CNN model

4. Compile and Train model: This step uses epochs of 30 epochs 
5. Evaluation dan test model

6. Implementation

After doing the above methodology obtained results that show the accuracy of the model is $60 \%$.

In the study [9] entitled "DEEP LEARNING BASED SAFE SOCIAL DISTANCING AND FACE MASK DETECTION IN PUBLIC AREAS FOR COVID19 SAFETY

GUIDELINES ADHERENCE". It combines MobileNetV2 lightweight neural network and single shot detector (SSD) by moving learning techniques to achieve a balance of resource limitations with accuracy in identification that can be used in real time to be able to monitor public places in detecting people who use masks or do not use masks and maintain a safe distance (social distancing). This study uses neural networking models to analyze the Real Time Streaming Protocol (RTSP) using OpenCV and MobileNetV2. This research also combines deep learning (Tensorflow) techniques with classic projective geometry techniques that provide good timeliness and high predictive accuracy, so that people will apply health protocols that use masks correctly and maintain distance. This research achieves an accuracy rate between $85 \%$ and $95 \%$. This research uses a dataset to be trained as many as 3,165 images divided into $80 \%$ to be trained which is classified by category using a mask and not using a mask and $20 \%$ others for testing, then the images will be done some pre- processing steps including images will be resize $224 \times 224$ pixels, and change the format. NumPy array. The process of training the model for 1000 steps using Adam optimizing algorithm, epochs (number of epoch $)=20$, batch size $(\mathrm{BS})=$

32. To monitor the social distance is used cv2 and after the person is identified it will then start with the coordinates bounding box and will do calculations around the person between the left and right part of the middle point (midpoint) and which becomes the midpoint is the person, and it will be ascertained that the ideal distance between people in the frame is in accordance with the difference. The testing stages of the model will detect everyone in the frame that is within the range of the camera and will be shown with a green bounding box on each person. If the person violates the safe distance from the social distance, then the box will change color to red and will simultaneously identify the person's face in the use of a mask or not using a mask. If the person does not wear a mask and does not maintain safe social distance then the system will give a warning to the competent authorities. This system is able to detect face mask and social distance with a precision score of $91.7 \%$ with a confidence score of 0.7 , precision value of 0.91 and recall value of 0.91 with FPS $=28.07$.

In the study [10] entitled "REAL TIME MULTI-SCALE FACIAL MASK DETECTION AND CLASSIFICATION USING DEEP TRANSFER LEARNING TECHNIQUES".

This study proposes two different multi-scale face mask

detection methods. The first method is face mask using YoloV3 (FMY3) and the second method is face mask using NASNetMobile (FMNMobile). These two approaches rely on CNN-based transfer learning which will take the pre-trained network model and modify the model according to needs. The FMY3 method uses yoloV3 object detection algorithm and uses a dataset of 680 types of face mask images with a ratio of $80 \%$ for training and $20 \%$ for testing of which there're 440 training images and 240 images for testing. This method gives 3 (three) categories and is given the label names Good, Bad and None. 'Good' indicates the person is wearing the mask correctly, 'Bad' indicates that the person is not wearing the mask, and 'None 'indicates that the person is not wearing the mask correctly. The FMNMobile method uses 1400 images divided into 2 (two) different folders namely face mask and without facemask. This method uses the Pre-trained Resnet- SSD100_caffee face detection model to detect faces from a given image input. The two methods produce good accuracy but FMNMobile is superior in accuracy rate with $98 \%$ recall compared to FMY3 with $91.7 \%$ recall.

\section{METHODOLOGY}

This research began with Data Gathering, Data sharing, data processing, Data Training, further model testing and analysis of test results.

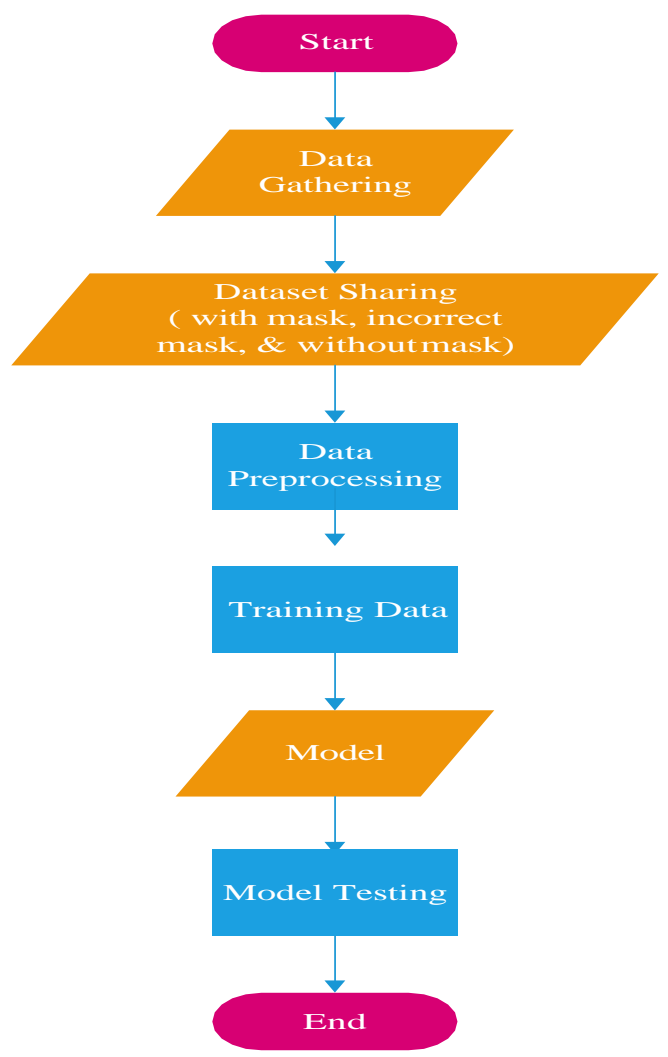

Figure 1 Research methodology The Right Face Mask Detection System

The research method conducted using MobileNetV2 is expected to improve performance in the detection of an object in real-time. This method also uses tensorflow framework because it is open source, easy to use due to easy coding and fast debugging.

\section{Data Collection}

Data collection is done by collecting photos of faces either using or not using masks, and collected from various sources such as kaggle. com [11] and Github [12]. Take a photo sample based on different facial poses. The photos of the face will be entered into the directory Dataset. The dataset will be carried out preprocesses such as the size of the photo data so as to produce more and varied data.

\section{Dataset Sharing}

This study divided the dataset into three, namely data with Mask, data incorrect Mask and data without Mask. For then data with Mask, data incorrect Mask and data without Mask is divided into $33.81 \%$ : $33.19 \%: 33.00 \%$. So, there are 2,079 datasets that are divided into, 703 facial photo data that uses masks incorrectly, 690 
facial photo data that use masks correctly, and 686 facial photo data that does not use masks. Data with Mask, data incorrect Mask and data without Mask is collected into each directory.

\section{Data Processing}

All data preprocesses to prepare data through the training process, data will perform the process of resizing and augmentation data. The purpose of this data process is to produce more varied data so that it can increase the level of accuracy.

\section{Stages of Training and Modeling}

\section{Data training}

Data training is done with the aim to get a model of the results of machine learning training. This model will be used for testing. This training process uses the MobileNetV2 model and Tensorflow framework which contains facial datasets that have been preprocessed before in order to learn and understand the information on the data by a machine or computer. In this training process there is a split dataset process, namely data train and data test with a ratio of $75 \%: 25 \%$, so in this split process will call the dataset directory that contains datasets that use masks and datasets that do not use masks. If the data train is able to learn the data well then there is a chance that the data train will be able to also learn the new data, namely data test. But conversely, if the data train is not able to learn the data well, then the data train will not be able to study the test data, where this condition will cause underfitting, underfitting is a low training accuracy and validation accuracy value and training loss and validation loss values that tend to be high.

Based on research from [13],said that the comparison of the number of epochs and steps per epoch affects the level of accuracy. The more epochs and steps per epoch used, the higher the level of accuracy obtained. In addition, the use of steps per epoch can also improve the accuracy obtained during the training process.

Therefore, in this training using epoch and Batch Size (BS) which have a value of 50 and 20 , respectively, with step per epoch 103 obtained from rounding the division of the number of epochs divided by batch size. The respective values of epoch, BS and step per epoch aim to get optimal results and avoid overfitting and underfitting, where these optimal results have a small error value.

After undergoing the training process, the graphic display of accuracy and loss can be seen by creating a model plot program or can be through tensor board. Plot model is a visualize tool using matplotlib which contains a pyplot package similar to the MATLAB interface. Tensor board is a visualize tool provided by TensorFlow.

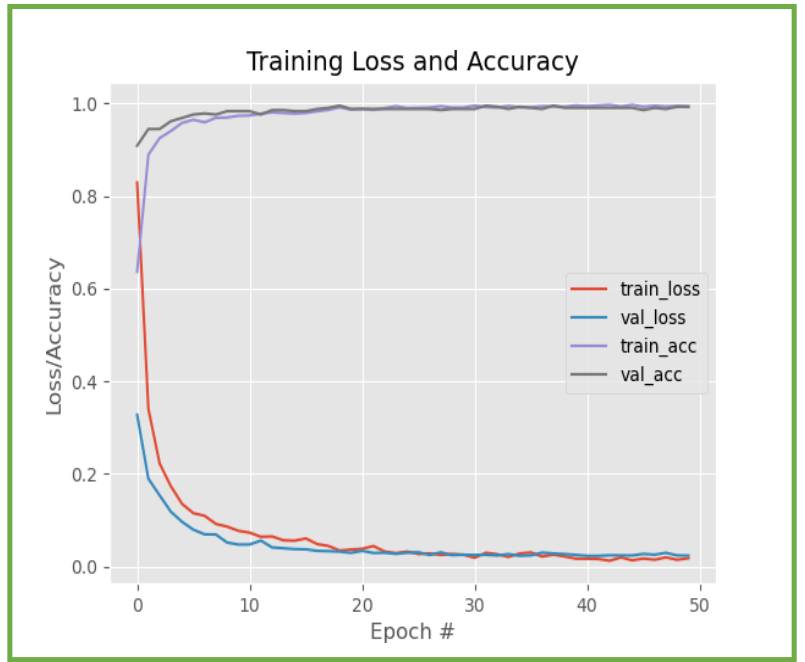

Figure 2 Training Loss and Accuracy

In the picture above it can be concluded that for train accuracy and validation accuracy for the initial Epoch that starts from above $80 \%$ until the final epoch is close to $100 \%$ or more precisely $99 \%$. As for train loss and validation loss at the initial epoch for train loss is at $40 \%$ and for validation loss is close to $20 \%$ or is at $18 \%$ until the final epoch for train loss and validation loss has dropped and is close to $0 \%$ or more precisely for train loss $2.1 \%$ and for validation loss of $5 \%$ for

20 epochs trained before. Epoch itself is when the entire dataset has gone through the training process on the Neural Network until it is returned to the beginning in one round. So that the training done before will do the training process as many as 20 times.

\section{Data training results model}

This data training model is the result of previous training and produces a model that will be used to predict or test the system. This model is saved by extension (.h5)

\section{Model Testing Preparation Stage}

The testing stage is the stage where the system that has been designed before and produces a model of training results that will be tested its ability with the aim of knowing whether the system can work properly as desired.

An algorithm in machine learning requires a training process and a testing process. The training process aims to train the algorithm in recognizing pre-prepared datasets and forming a model based on that training. While in the testing process aims to test a model formed in the training process to find out how it performs and performs. Here is the preparation of the testing process on this research. 

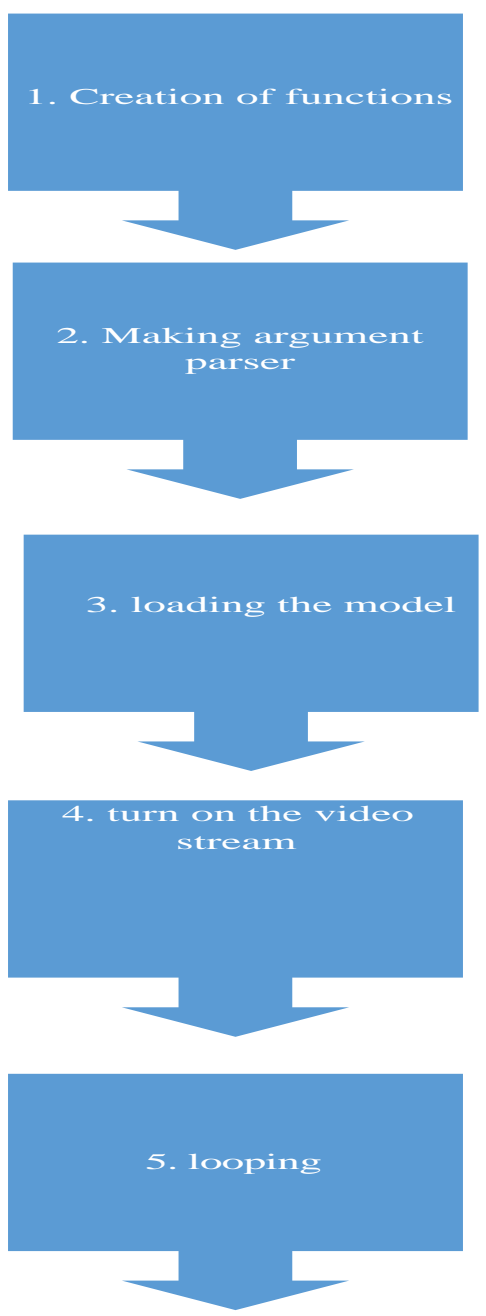

Figure 3 Block Diagram of The Model Testing Preparation Stage

\section{The First Step}

The first step is to create a function using python programming language that aims to detect and predict the mask on each face using a mask that appears and provides a frame (bounding box) on each face that is detected and predicted.

\section{Step Two}

The second step is to create an argument parser to run triggers with different actions according to the user's wishes. In the argument parser there are 2 argument parser that have been given the default value, namely in the argument face detector which aims to load the face detector model so that it can detect a person's face and then on the model mask detector that has been trained before aimed at detecting the use of masks or not using masks.

\section{Step Three}

Step Three is to load the model to detect the face and the model to detect the use of the right mask or not to use the mask by using a parser argument that has been made before.

$$
\text { 4. Step Four }
$$

The fourth step is to turn on the video stream in this case is to use the webcam camera on the laptop.
5. Step Five

The fifth step is to do a loop that aims to

a. Capture frames from video streams and resize frames with a maximum of 400 pixels

b. Calling a previously created function called "detect_and_predict_mask" that aims to detect faces on frames and detect the use of masks on frames.

c. Determine the location of the face and give a bounding box and label and color used to distinguish using the right mask or using a mask that is not appropriate or does not use a mask by labeling the mask, incorrect mask, and without mask and giving green color to use the mask the right color, yellow to use the mask that is not right and the color red not to use the mask

d. Provide a percentage to show the level of accuracy of the system in identifying the right use of masks or improper use

of masks or not using masks.

\section{RESULTS AND DISCUSSIONS}

Testing of the model that has been produced, tested with several scenarios that have been created by the researcher, namely:

\section{Testing the system under the right conditions of use of the mask}

Mask detection testing is done to ensure that the system can detect objects with multiple parameters. These parameters are:

- Objects use masks properly and precisely

- The system is tested with different object distance conditions

- The system is tested with different object angle conditions

Below is the test table:

Table 1 System Test Results in detecting the right use of masks

\begin{tabular}{|l|l|l|l|}
\hline Parameters & $\begin{array}{l}\text { Distance } \\
(\mathrm{cm})\end{array}$ & Information & Percentage \\
\hline \multirow{3}{*}{ Perpendicular } & $50 \mathrm{~cm}$ & Detected & $96,26 \%$ \\
\cline { 2 - 4 } & $100 \mathrm{~cm}$ & Detected & $96,06 \%$ \\
\cline { 2 - 4 } & $160 \mathrm{~cm}$ & Detected & $94,01 \%$ \\
\hline \multirow{2}{*}{$\begin{array}{l}30-\text {-degree } \\
\text { rotation } \\
\text { the right }\end{array}$} & $50 \mathrm{~cm}$ & Detected & $99,96 \%$ \\
\cline { 2 - 4 } $\begin{array}{l}\text { Rotation } \\
\text { degrees to the } \\
\text { right }\end{array}$ & $100 \mathrm{~cm}$ & Detected & $99,95 \%$ \\
\cline { 2 - 4 } & $160 \mathrm{~cm}$ & Detected & $92,40 \%$ \\
\cline { 2 - 4 } $\begin{array}{l}30-\text {-degree } \\
\text { rotation } \\
\text { the left }\end{array}$ & $100 \mathrm{~cm}$ & Detected & $51,81 \%$ \\
\cline { 2 - 4 } & $160 \mathrm{~cm}$ & Detected & $76,96 \%$ \\
\cline { 2 - 4 } $\begin{array}{l}\text { Rotation } 90 \\
\text { degrees to the }\end{array}$ & $100 \mathrm{~cm}$ & Detected & $98,39 \%$ \\
\cline { 2 - 4 } left & $160 \mathrm{~cm}$ & Detected & $98,92 \%$ \\
\cline { 2 - 4 } & $50 \mathrm{~cm}$ & Detected & $97,40 \%$ \\
\cline { 2 - 4 } & $100 \mathrm{~cm}$ & Detected & $52,44 \%$ \\
\hline
\end{tabular}


The percentage shown by the system identifies the degree of accuracy. All percentages exceeded $50 \%$ with an average of $85.906 \%$ and the green Bounding Box identified the proper use of masks according to the rules, indicating the system can operate properly and properly.

\section{Testing the system under improper \\ use of masks}

Mask detection testing is done to ensure that the system can detect objects with multiple parameters. These parameters are:

- Objects use masks incorrectly

- The system is tested with different object distance conditions

- The system is tested with different object angle conditions

Below are the conditions of improper use of the mask based on the above parameters:

Testing is done in the condition that the mask does not cover the nose area but the mask closes the mouth area.

Below is the test table:

Table 2 The test results in the condition that the mask does not cover the nose area but the mask covers the mouth area

\begin{tabular}{|l|l|l|l|}
\hline Parameters & $\begin{array}{l}\text { Distance } \\
(\mathrm{cm})\end{array}$ & Information & Percentage \\
\hline \multirow{3}{*}{ Perpendicular } & $50 \mathrm{~cm}$ & Detected & $98,90 \%$ \\
\cline { 2 - 4 } & $100 \mathrm{~cm}$ & Detected & $81,42 \%$ \\
\cline { 2 - 4 } & $160 \mathrm{~cm}$ & Detected & $98,55 \%$ \\
\hline \multirow{2}{*}{$\begin{array}{l}\text { 30-degree } \\
\text { rotation } \\
\text { the right }\end{array}$} & $50 \mathrm{~cm}$ & Detected & $59,45 \%$ \\
\hline & $100 \mathrm{~cm}$ & Detected & $95,47 \%$ \\
\cline { 2 - 4 } $\begin{array}{l}\text { Rotation } \\
\text { degrees to the } \\
\text { right }\end{array}$ & $160 \mathrm{~cm}$ & Detected & $81,60 \%$ \\
\hline \multirow{3}{*}{$\begin{array}{l}\text { 30-degree } \\
\text { rotation } \\
\text { the left }\end{array}$} & $100 \mathrm{~cm}$ & Detected & $80,11 \%$ \\
\hline & $160 \mathrm{~cm}$ & Detected & $82,47 \%$ \\
\hline \multirow{2}{*}{$\begin{array}{l}\text { Rotation } \\
\text { degrees to the } \\
\text { left }\end{array}$} & $50 \mathrm{~cm}$ & Detected & $72,33 \%$ \\
\cline { 2 - 4 } & $100 \mathrm{~cm}$ & Detected & $76,70 \%$ \\
\cline { 2 - 4 } & $160 \mathrm{~cm}$ & Detected & $75,56 \%$ \\
\cline { 2 - 4 } & $100 \mathrm{~cm}$ & Detected & $95,96 \%$ \\
\hline
\end{tabular}

The percentage shown by the system identifies the degree of accuracy. All percentages exceeded $50 \%$ with an average of $83.62 \%$ and the yellow Bounding Box identified the use of masks that are not in accordance with the rules, this indicates the system can operate properly and appropriately.

\section{Testing is done in the condition that the}

mask does not cover the mouth area but

the mask covers the nose area.

Below is the test table:

Table 3 The test results are carried out in the condition that the mask covers the mouth area but the mask covers the nose area

\begin{tabular}{|l|l|l|l|}
\hline Parameters & $\begin{array}{l}\text { Distance } \\
(\mathrm{cm})\end{array}$ & Information & Percentage \\
\hline \multirow{3}{*}{ Perpendicular } & $50 \mathrm{~cm}$ & Detected & $58,54 \%$ \\
\cline { 2 - 4 } & $100 \mathrm{~cm}$ & Detected & $55,52 \%$ \\
\cline { 2 - 4 } & $160 \mathrm{~cm}$ & Detected & $65,56 \%$ \\
\hline
\end{tabular}

\begin{tabular}{|c|c|c|c|}
\hline \multirow{3}{*}{$\begin{array}{l}\text { 30-degree } \\
\text { rotation } \\
\text { the right }\end{array}$} & $50 \mathrm{~cm}$ & Detected & $94,01 \%$ \\
\hline & $100 \mathrm{~cm}$ & Detected & $96,22 \%$ \\
\hline & $160 \mathrm{~cm}$ & Detected & $85,59 \%$ \\
\hline \multirow{3}{*}{$\begin{array}{l}\text { Rotation } 90 \\
\text { degrees to the } \\
\text { right }\end{array}$} & $50 \mathrm{~cm}$ & Detected & $95,98 \%$ \\
\hline & $100 \mathrm{~cm}$ & Detected & $93,65 \%$ \\
\hline & $160 \mathrm{~cm}$ & Detected & $89,92 \%$ \\
\hline \multirow{3}{*}{$\begin{array}{l}\text { 30-degree } \\
\text { rotation to } \\
\text { the left }\end{array}$} & $50 \mathrm{~cm}$ & Detected & $97,78 \%$ \\
\hline & $100 \mathrm{~cm}$ & Detected & $78,45 \%$ \\
\hline & $160 \mathrm{~cm}$ & Detected & $52,08 \%$ \\
\hline \multirow{3}{*}{$\begin{array}{l}\text { Rotation } 90 \\
\text { degrees to the } \\
\text { left }\end{array}$} & $50 \mathrm{~cm}$ & Detected & $75,56 \%$ \\
\hline & $100 \mathrm{~cm}$ & Detected & $94,40 \%$ \\
\hline & $160 \mathrm{~cm}$ & Detected & $96,78 \%$ \\
\hline
\end{tabular}

The percentage shown by the system identifies the degree of accuracy. All percentages exceeded 52\% with an average of $82 \%$ and the yellow Bounding Box identified the use of masks that were not in accordance with the rules, this indicates the system can operate properly and appropriately.

Testing is done in the condition that the mask does not cover the nose area and mouth area

The test is done in two (two) ways:

1. Mask closes eyes and forehead

2. Mask covers the chin area

only Below is the test table:

Table 4 The test results were carried out in the condition that the mask did not cover the nose area and mouth area

\begin{tabular}{|c|c|c|c|}
\hline Parameters & $\begin{array}{l}\text { Distance } \\
(\mathrm{cm})\end{array}$ & Information & Percentage \\
\hline \multirow{6}{*}{ Perpendicular } & \multirow{2}{*}{$50 \mathrm{~cm}$} & Detected & $78,55 \%$ \\
\hline & & Detected & $99,77 \%$ \\
\hline & \multirow[t]{2}{*}{$100 \mathrm{~cm}$} & $\begin{array}{l}\text { Detected but } \\
\text { not in } \\
\text { accordance } \\
\text { with system } \\
\text { settings }\end{array}$ & $98,89 \%$ \\
\hline & & Detected & $88,71 \%$ \\
\hline & \multirow[t]{2}{*}{$160 \mathrm{~cm}$} & $\begin{array}{l}\text { Detected but } \\
\text { not in } \\
\text { accordance } \\
\text { with system } \\
\text { settings }\end{array}$ & $95,34 \%$ \\
\hline & & Detected & $88,23 \%$ \\
\hline \multirow{7}{*}{$\begin{array}{l}\text { 30-degree } \\
\text { rotation to } \\
\text { the right }\end{array}$} & \multirow{2}{*}{$50 \mathrm{~cm}$} & Detected & $70,91 \%$ \\
\hline & & Detected & $90,15 \%$ \\
\hline & $100 \mathrm{~cm}$ & Detected & $71,72 \%$ \\
\hline & & Detected & $95,97 \%$ \\
\hline & \multirow[t]{2}{*}{$160 \mathrm{~cm}$} & $\begin{array}{l}\text { Detected but } \\
\text { not in } \\
\text { accordance } \\
\text { with system } \\
\text { settings }\end{array}$ & $99,03 \%$ \\
\hline & & Detected & $89,01 \%$ \\
\hline & $50 \mathrm{~cm}$ & $\begin{array}{l}\text { Detected but } \\
\text { not precisely } \\
\text { in accordance } \\
\text { with system } \\
\text { settings }\end{array}$ & $99,58 \%$ \\
\hline
\end{tabular}




\begin{tabular}{|c|c|c|c|}
\hline \multirow{5}{*}{$\begin{array}{l}\text { Rotation } 90 \\
\text { degrees to the } \\
\text { right }\end{array}$} & & Detected & $95,21 \%$ \\
\hline & $100 \mathrm{~cm}$ & Detected & $80,28 \%$ \\
\hline & & Detected & $92,11 \%$ \\
\hline & $160 \mathrm{~cm}$ & $\begin{array}{l}\text { Detected but } \\
\text { not in } \\
\text { accordance } \\
\text { with system } \\
\text { settings }\end{array}$ & $95,10 \%$ \\
\hline & & Detected & $94,03 \%$ \\
\hline \multirow{6}{*}{$\begin{array}{l}30 \text {-degree } \\
\text { rotation to } \\
\text { the left }\end{array}$} & $50 \mathrm{~cm}$ & Detected & $74,20 \%$ \\
\hline & & Detected & $97,04 \%$ \\
\hline & $100 \mathrm{~cm}$ & Detected & $67,51 \%$ \\
\hline & & Detected & $82,55 \%$ \\
\hline & $160 \mathrm{~cm}$ & Detected & $58,92 \%$ \\
\hline & & Detected & $92,94 \%$ \\
\hline \multirow{6}{*}{$\begin{array}{l}\text { Rotation } 90 \\
\text { degrees to the } \\
\text { left }\end{array}$} & $50 \mathrm{~cm}$ & $\begin{array}{l}\text { Detected but } \\
\text { not in } \\
\text { accordance } \\
\text { with system } \\
\text { settings }\end{array}$ & $50,67 \%$ \\
\hline & & Detected & $95,49 \%$ \\
\hline & $100 \mathrm{~cm}$ & $\begin{array}{l}\text { Detected but } \\
\text { not in } \\
\text { accordance } \\
\text { with system } \\
\text { settings }\end{array}$ & $95,18 \%$ \\
\hline & & Detected & $97,25 \%$ \\
\hline & $160 \mathrm{~cm}$ & Detected & $44,57 \%$ \\
\hline & & Detected & $71,07 \%$ \\
\hline
\end{tabular}

Conducted in the first and second way, 23 successful tests with an average of $76.67 \%$ and 7 tests failed with an average of $23.33 \%$. 7 tests that failed to identify the wearing of masks were caused as follows:

- Lack of number and variety of datasets covering the eye area and forehead area only.

- 8 successful tests in the first test have similarities with the dataset so that the system can identify them.

- Meanwhile, 7 tests that failed in the first test did not have similarities with the dataset both in distance and angle so that the system failed to identify.

Testing system in the condition of not

\section{using a mask}

Mask detection testing is done to ensure that the system can detect objects with multiple parameters. These parameters are:

- Objects do not use masks

- The system is tested with different object distance conditions

- The system is tested with different object angle conditions

Below is the test table:
Table 5 Test results in the condition of not using a mask

\begin{tabular}{|c|c|c|c|}
\hline Parameters & Distance $(\mathrm{cm})$ & Information & Percentage \\
\hline \multirow{3}{*}{ Perpendicular } & $50 \mathrm{~cm}$ & Detected & $100 \%$ \\
\hline & $100 \mathrm{~cm}$ & Detected & $100 \%$ \\
\hline & $160 \mathrm{~cm}$ & Detected & $100 \%$ \\
\hline \multirow{3}{*}{$\begin{array}{l}\text { 30-degree } \\
\text { rotation } \\
\text { the right }\end{array}$} & $50 \mathrm{~cm}$ & Detected & $99,95 \%$ \\
\hline & $100 \mathrm{~cm}$ & Detected & $99,95 \%$ \\
\hline & $160 \mathrm{~cm}$ & Detected & $99,04 \%$ \\
\hline \multirow{3}{*}{$\begin{array}{l}\text { Rotation } 90 \\
\text { degrees to the } \\
\text { right }\end{array}$} & $50 \mathrm{~cm}$ & Detected & $100 \%$ \\
\hline & $100 \mathrm{~cm}$ & Detected & $99,95 \%$ \\
\hline & $160 \mathrm{~cm}$ & Detected & $99,97 \%$ \\
\hline \multirow{3}{*}{$\begin{array}{l}30 \text {-degree } \\
\text { rotation } \\
\text { the left }\end{array}$} & $50 \mathrm{~cm}$ & Detected & $100 \%$ \\
\hline & $100 \mathrm{~cm}$ & Detected & $100 \%$ \\
\hline & $160 \mathrm{~cm}$ & Detected & $100 \%$ \\
\hline \multirow{3}{*}{$\begin{array}{l}\text { Rotation } 90 \\
\text { degrees to the } \\
\text { left }\end{array}$} & $50 \mathrm{~cm}$ & Detected & $100 \%$ \\
\hline & $100 \mathrm{~cm}$ & Detected & $99,96 \%$ \\
\hline & $160 \mathrm{~cm}$ & Detected & $99,93 \%$ \\
\hline
\end{tabular}

For each way done in the test get varying results, both percentage values and results from the system in identifying the use of the mask. In the first way, the mask closed the eye area and forehead area and left the nose area and mouth area open, getting results from 15 tests, 8 tests were successful in identifying the use of masks, and 7 other tests failed in identifying the use of the mask. While in the second way, the mask only closed the chin area and left the nose area and mouth area open, getting the results of 15 successful tests in identifying the use of the mask. So, there were 30 test results

The percentage shown by the system identifies the degree of accuracy. All percentages exceeded $99 \%$ with an average of 99.92\% and the red Bounding Box identified the object not using a mask, indicating the system can operate properly and precisely.

\section{CONCLUSION}

Based on the tests and discussions that have been done, this research can be concluded that the system can detect and identify in the correct use of masks based on the applicable kemenkes.go.id.

The process of identifying the correct use of masks in this system uses deep learning methods with MobileNetV2 modules and Tensorflow framework. The stages of this research process include Data Collection, Dataset Sharing,

Data Processing, and System Design (Preparation Stage, Training and Modeling Stage, and Model Testing Stage)

Based on 90 tests conducted results obtained there are 7 invalid tests, so the accuracy of the system that has been built has an average of $86.95 \%$.

Based on these results, the research can be improved again, such as increasing the scope of the research by giving a warning vote or being able to directly impose fines or penalties for those who violate health protocols, by means of a computer that can recognize the user's face and synchronize it with the community ID card database.

\section{ACKNOWLEDGMENTS}

Our thanks to the experts who have contributed.

\section{REFERENCES}

[1] S. Chavez, B. Long, A. Koyfman, and S. Y. Liang, "Coronavirus Disease (COVID-19): A primer for emergency physicians," Am. J. Emerg. Med., vol. 44, pp. 220-229, Jun. 
2021, doi: 10.1016/j.ajem.2020.03.036.

[2] "WHO Coronavirus (COVID-19) Dashboard | WHO Coronavirus (COVID-19) Dashboard With Vaccination Data." https://covid19.who.int/ (accessed Jun. 24, 2021).

[3] S. W. Sim, K. S. P. Moey, and N. C. Tan, "The use of facemasks to prevent respiratory infection: A literature review in the context of the Health Belief Model," Singapore Med. J., vol. 55, no. 3, pp. 160- 167, 2014, doi: 10.11622/smedj.2014037.

[4] B. J. Cowling et al., "Facemasks and hand hygiene to prevent influenza transmission in households: A cluster randomized trial," Annals of Internal Medicine, Oct. 06, 2009. https://www.acpjournals.org/doi/pdf/10.7326/00034819-151-7-200910060-00142 (accessed Jun. 24, 2021).

[5] S. M. Tracht, S. Y. Del Valle, and J. M. Hyman, "Mathematical modeling of the effectiveness of facemasks in reducing the spread of novel influenza a (H1N1)," PLoS One, vol. 5, no. 2, p. 9018, Feb. 2010, doi: 10.1371/journal.pone.0009018.

[6] T. Jefferson et al., "Physical interventions to interrupt or reduce the spread of respiratory viruses," Cochrane Database of Systematic Reviews, Nov. 20, 2020. https://www.cochranelibrary.com/cdsr/doi/10.1002/1 4651858.CD006207.pub5/full (accessed Jun. 24, 2021).

[7] “Cara Memakai Masker yang Benar,” Feb. 04, 2020. https://promkes.kemkes.go.id/cara-memakai-maskeryang-benar (accessed Oct. 09, 2021).

[8] D. Ullala Mata, "FACE MASK DETECTION USING
CONVOLUTIONAL NEURAL NETWORK," J. Nat. Remedies, vol. 21, no. 12, pp. 14-19, Apr. 2021, Accessed: Jun. 29, 2021. [Online]. Available: http://www.jnronline.com/ojs/index.php/about/article/view/7 84.

[9] S. Yadav, "Deep Learning based Safe Social Distancing and Face Mask Detection in Public Areas for COVID-19 Safety Guidelines Adherence," Int. J. Res. Appl. Sci. Eng. Technol., vol. 8, no. 7, pp. 1368- 1375, Jul. 2020, doi: 10.22214/ijraset.2020.30560

[10] S. K. Addagarla, G. Kalyan Chakravarthi, and P. Anitha, "Real time multi-scale facial mask detection and classification using deep transfer learning techniques," Int. $J$. Adv. Trends Comput. Sci. Eng., vol. 9, no. 4, pp. 4402-4408, 2020, doi: 10.30534/ijatcse/2020/33942020.

[11] O. Gurav, "Face Mask Detection Dataset | Kaggle." https://www.kaggle.com/omkargurav/face-mask- dataset (accessed Jul. 12, 2021).

[12] "GitHub - X-zhangyang/Real-World-Masked-Face- Dataset: Real-World Masked Face Dataset，罩人脸数据集.” https://github.com/X- zhangyang/Real-World-Masked-FaceDataset (accessed Jul. 12, 2021).

[13] E. Yuliani, A. N. Aini, and C. U. Khasanah, "Perbandingan Jumlah Epoch Dan Steps Per Epoch Pada Convolutional Neural Network Untuk Meningkatkan Akurasi Dalam Klasifikasi Gambar," J. Inf. J. Penelit. dan Pengabdi. Masy., vol. 5, no. 3, pp. 23-27, Jan. 2019, doi: 10.46808/INFORMA.V5I3.140. 